\section{How effective is macula-off retinal detachment surgery. Might good outcome be predicted?}

\begin{abstract}
Purpose To determine factors associated with anatomical and functional outcomes of macula-off retinal detachment surgery in a modern vitreoretinal unit.
\end{abstract}

Methods A retrospective casenote review of 185 patients presenting with macula-off retinal detachment was performed. Demographic and ocular characteristics were determined. Logistic regression analysis was used to determine (1) the effect of these factors on

'St Thomas' Hospital, Lambeth Palace Road, London, SE1 7EH, UK

${ }^{2}$ Research \& Development, Moorfields Eye Hospital, City Road, London EC1V 2PD, UK

Correspondence: E Doyle, Thomas' Hospital, Lambeth Palace Road, London, SE1 7EH, UK

Tel: + 4477994 48543;

Fax: + 448714338914

E-mail: edrachie@

btinternet.com

Received: 25 October 2005 Accepted in revised form: 5 December 2005

Published online: 3 February 2006

E Doyle had full access to all the data in the study and takes responsibility for the integrity of the data and the accuracy of the data analysis

Disclaimer: The authors have no commercial interests in any products mentioned in this article
E Doyle ${ }^{1}$, EN Herbert ${ }^{1}$, C Bunce ${ }^{2}$, TH Williamson' and DAH Laidlaw'

and duration of detachment also affect outcomes.

Eye (2007) 21, 534-540. doi:10.1038/sj.eye.6702260; published online 3 February 2006

Keywords: retinal detachment; outcome; eye surgery; macula; visual outcome

Introduction

The primary anatomical success rate of surgery for retinal detachment can be high and should be in the range of $80-90 \% .{ }^{1,2}$ Failure to achieve a primary anatomical success (retinal reattachment with one operation) reduces the chance of a good visual outcome. ${ }^{3}$ However, for retinal detachments with macular detachment primary anatomical success does not necessarily achieve a good visual outcome and the functional results vary greatly. The reasons for poor visual acuity cannot always be determined and previous studies relate mainly to the outcomes for patients who have undergone 'conventional' external scleral buckling surgery with or without external drainage. In recent years, a shift towards the increasing use of primary internal pars plana vitrectomy away from these conventional buckling techniques has been seen. ${ }^{4}$ This study of the surgical management of macula-off retinal detachments determines anatomical and visual outcomes and attempts to determine which preoperative factors influenced these outcomes in the setting of a modern vitreoretinal unit.

\section{Materials and methods}

A retrospective casenote review was carried out of 185 patients presenting with primary 
macula-off retinal detachment. Macular detachment was defined as detachment involving the fovea with any resulting loss of central Snellen visual acuity. The series was an unbiased selection of cases under the care of two consultants (THW: $n=110$ and DAHL: $n=75$ ) in a vitreoretinal unit of a teaching hospital. Trainees performed 93 of the 185 operations. The operations were carried out between 13/11/98 and 10/2/01 and followup was 4 months to 2.5 years. (median 7.9 months).

Primary anatomical success was defined as a reattached retina after one procedure and remaining attached to final follow-up. Ultimate anatomical success included primary anatomical success as well as those patients for whom a reattached retina was achieved with subsequent surgery. Patients whose operations involved the use of silicone oil were not considered to be primary or ultimate successes unless oil was subsequently removed without redetachment. Visual acuity (best with correction or pinhole) was converted from Snellen acuity to $\log$ MAR to allow statistical analysis. So, for example, Snellen acuities of $6 / 6$ and $6 / 12$ would have a logMAR of 0 and 0.3 , respectively. In cases postoperatively where visual acuity was limited by cataract, the best visual acuity before development of the cataract was used instead of final visual acuity as the visual outcome. Demographic and ocular characteristics were determined. Univariate and multiple variable logistic regression were used to assess the evidence of associations between (1) primary anatomical success (2) ultimate anatomical success and (3) visual outcome and each of age, duration of macular detachment, preoperative visual acuity, extent of retinal detachment (in quadrants), grade of surgeon, lens status (ie if the patients were phakic, pseudophakic or aphakic) and presence of grade $\mathrm{C}$ proliferative vitreoretinopathy (PVR). When considering visual outcome, analysis was restricted to subjects with no prior reason for poor vision such as macular degeneration, diabetic retinopathy, or retinal vascular occlusion. Factors significant at the 0.2 level in the univariate model were included in the multiple variable regression. Refractive error was not sufficiently well documented to be included in the analysis and is confused by the presence of various types of lens status.

\section{Results (see Tables 1-3)}

\section{Demography - see Table 1}

The median age of the patients was 63 years (range 17-91). In all, 39\% of patients had been macula-off for less than 1 week with 37\% macula-off between 1 week and 30 days and $24 \%$ greater than 30 days. Phakic patients comprised $74 \%$ of patients with the rest being
Table 1 Demographic data describing the 185 patients in the study

\begin{tabular}{|c|c|c|}
\hline Factor & Number & $\%$ of available data \\
\hline \multicolumn{3}{|l|}{ Sex } \\
\hline Female & 69 & 38 \\
\hline Male & 116 & 62 \\
\hline \multicolumn{3}{|c|}{ Duration of detachment } \\
\hline$<7$ days & 64 & 39 \\
\hline $7-<30$ days & 60 & 37 \\
\hline$\geq 30$ days & 39 & 24 \\
\hline \multicolumn{3}{|l|}{ Grade of surgeon } \\
\hline Consultant & 92 & 50 \\
\hline Fellow & 93 & 50 \\
\hline \multicolumn{3}{|l|}{ Phakic } \\
\hline No & 48 & 26 \\
\hline Yes & 137 & 74 \\
\hline \multicolumn{3}{|l|}{$P V R$ grade $c$} \\
\hline No & 165 & 89 \\
\hline Yes & 20 & 11 \\
\hline \multicolumn{3}{|l|}{ No. of breaks } \\
\hline$\leq 1$ & 87 & 50 \\
\hline 2 & 44 & 25 \\
\hline$>2$ & 42 & 24 \\
\hline \multicolumn{3}{|l|}{ No of quadrants } \\
\hline$\leq 2$ & 88 & 51 \\
\hline 3 & 50 & 29 \\
\hline \multirow[t]{2}{*}{4} & 36 & 21 \\
\hline & Median & (Interquartile range) \\
\hline Age (years) & 63 & $(54,70)$ \\
\hline
\end{tabular}

pseudophakic with the exception of two aphakes. PVR grade $\mathrm{C}$ was present to some degree in $11 \%$. The extent of detachment was 2 quadrants or less, 3 and 4 quadrants in 51,29 and $21 \%$ of patients, respectively.

\section{Primary anatomical success - see Tables 2 and 3 for odds ratios}

Primary anatomical success was defined as remaining attached after one detachment procedure and to final follow-up without continued oil tamponade. The primary anatomical success was $76 \%$. However, 10 patients had flat retinas under oil that were not considered to be primary successes. If these retinas were to remain attached after removal of the oil at a later date they would meet our criteria for primary success potentially raising the primary success rate to $81.6 \%$.

With multivariate analysis statistically significant factors predicting primary anatomical success were preoperative logMAR visual acuity $(P=0.01)$, preoperative PVR $(P=0.03)$ and the number of breaks. 
Table 2 Results - odds ratios (with 95\% confidence intervals) for each factor in relation to primary anatomical success, final anatomical success, and visual outcome

\begin{tabular}{|c|c|c|c|}
\hline Factor & Primary anatomical success & Final anatomical success & Visual outcome \\
\hline Age (per year) & $0.997(0.97,1.02)$ & $0.98(0.95,1.01)$ & $0.98(0.95,1.00)^{*}$ \\
\hline \multicolumn{4}{|l|}{$\operatorname{Sex}$} \\
\hline Female & 1 & 1 & 1 \\
\hline Male & $0.78(0.38,1.58)$ & $0.8(0.34,1.89)$ & $0.96(0.51,1.80)$ \\
\hline \multicolumn{4}{|c|}{ Duration of detachment } \\
\hline$<7$ days & 1 & 1 & 1 \\
\hline $7-<30$ days & $1.02(0.42,2.45)$ & $0.73(0.19,2.87)$ & $0.51(0.24,1.08)$ \\
\hline$\geq 30$ days & $0.51(0.21,1.26)$ & $0.15(0.04,0.51)^{*}$ & $0.35(0.14,0.84)^{*}$ \\
\hline Preop VA (per unit) & $0.24(0.1,0.61)^{*}$ & $0.09(0.02,0.44)^{*}$ & $0.21(0.10,0.41)^{*}$ \\
\hline \multicolumn{4}{|l|}{ Grade of surgeon } \\
\hline Consultant & 1 & 1 & 1 \\
\hline Fellow & $0.96(0.49,1.87)$ & $0.93(0.41,2.10)$ & $0.45(0.24,0.83)^{*}$ \\
\hline \multicolumn{4}{|l|}{ Phakic } \\
\hline No & 1 & 1 & 1 \\
\hline Yes & $0.77(0.35,1.70)$ & $1.86(0.78,4.40)$ & $0.87(0.43,1.73)$ \\
\hline \multicolumn{4}{|l|}{$P V R$ grade $c$} \\
\hline No & 1 & 1 & 1 \\
\hline Yes & $0.27(0.10,0.70)^{*}$ & $0.15(0.05,0.41)^{*}$ & $0.41(0.14,1.21)$ \\
\hline \multicolumn{4}{|l|}{ No. of breaks } \\
\hline$\leq 1$ & 1 & 1 & 1 \\
\hline 2 & $0.33(0.15,0.78)^{*}$ & $0.65(0.24,1.76)$ & $0.60(0.28,1.30)$ \\
\hline$>2$ & $0.54(0.22,1.32)$ & $1.07(0.35,3.31)$ & $0.81(0.37,1.77)$ \\
\hline \multicolumn{4}{|l|}{ No. of quadrants } \\
\hline$\leq 2$ & 1 & 1 & 1 \\
\hline 3 & $0.61(0.27,1.39)$ & $0.33(0.11,1.00)^{*}$ & $0.73(0.35,1.49)$ \\
\hline 4 & $0.42(0.18,1.00)$ & $0.16(0.06,0.49)^{*}$ & $0.34(0.14,0.87)^{*}$ \\
\hline
\end{tabular}

Factors with significance at the $<0.05$ level are shown in italics with asterisks. For the visual outcome analysis patients with pre-existing reasons for poor vision were excluded. The results presented for visual outcome univariate. Multivariate analysis to detect independently associated factors are presented in the text.

The primary anatomical success achieved for patients with preoperative visual acuities of $6 / 18$ or better, $6 / 24-6 / 60$ and less than $6 / 60$ were $92.9,89$, and $70.7 \%$ respectively. The potential primary success rates for these three groups allowing for future removal of silicone oil were $92.9,91.7$ and $77.7 \%$. For patients with preoperative PVR grade C primary success was $55 \%(n=20)$. Patients with two breaks or three or more breaks had lower primary success rates $(50 \% ; P=0.003$ and $71 \% ; P=0.036$, respectively) than those where one or no breaks were found $(83 \%)$. In nine patients no break was found but in eight of these primary success was nonetheless achieved.

Fellows and consultants had similar primary success rates $(76 \% ; n=93$ vs $75 \% ; n=92)$.

There were seven missed/new breaks as a cause of failure for trainees versus one for consultants. Other causes of primary failure were continued oil tamponade
(10 patients), unsupported breaks (eight patients), PVR (nine patients), and the cause of primary failure could not be determined in seven patients.

\section{Ultimate anatomical success}

Ultimate anatomical success was $84 \%$. There were 14 patients not considered to be ultimate successes because the eyes still contained oil at final follow-up. Hence, the result would have risen to $92 \%$ if these patients were included.

With a multiple variable analysis only preoperative $\log$ MAR visual acuity and duration of macular detachment achieved significance ( $P=0.03$ in both cases), although with the initial univariate analysis number of detached quadrants at presentation (worse ultimate success for 3 or 4 quadrants detached) and presence of 
PVR grade $\mathrm{C}$ at presentation had also shown significance at the 0.05 level.

The ultimate anatomical success achieved for patients with preoperative visual acuities of $6 / 18$ or better, 6/24-6/60 and less than $6 / 60$ were $100 \%, 97.2$ and $80.8 \%$, respectively. The figures would rise to 100,100 , and $90.8 \%$ considering the potential for success of those patients with oil still in the eye. Patients with duration of detachment greater than 30 days had an ultimate success of $72 \%$. For patients with PVR the ultimate success was $75 \%(n=20)$. Patients with 2 or less quadrants of detached retina had an ultimate success of $93 \%$ and those with 3 or 4 quadrants of detachment had ultimate success of $77 \%$.

\section{Visual outcome}

For all patients together $44 \%$ regained $6 / 12$ Snellen or better, $33 \%$ had a final vision worse than $6 / 24$, the

Table 3 Results of multiple variable analysis - Odds ratios (with 95\% confidence intervals)

\begin{tabular}{|c|c|c|c|}
\hline Factor & $\begin{array}{l}\text { Primary } \\
\text { anatomical } \\
\text { success }\end{array}$ & $\begin{array}{c}\text { Final } \\
\text { anatomical } \\
\text { success }\end{array}$ & $\begin{array}{c}\text { Visual } \\
\text { outcome }\end{array}$ \\
\hline \multicolumn{4}{|c|}{ Duration of detachment } \\
\hline$<7$ days & & 1 & \\
\hline $7-30$ days & & $0.75(0.18,3.17)$ & \\
\hline$\geq 30$ days & & $0.22(0.06,0.88)$ & \\
\hline Preop VA (per unit) & $\begin{array}{c}0.23 \\
(0.07,0.70)\end{array}$ & $0.13(0.02,0.84)$ & $\begin{array}{c}0.20 \\
(0.08,0.51)\end{array}$ \\
\hline $\begin{array}{l}\text { Grade of surgeon } \\
\text { Consultant } \\
\text { Fellow }\end{array}$ & & & \\
\hline \multicolumn{4}{|l|}{ PVR grade $c$} \\
\hline No & 1 & & \\
\hline Yes & $0.28(0.09,0.89)$ & & \\
\hline \multicolumn{4}{|l|}{ No. of breaks } \\
\hline$\leq 1$ & 1 & & \\
\hline 2 & $0.22(0.08,0.60)$ & & \\
\hline$>2$ & $0.33(0.12,0.93)$ & & \\
\hline
\end{tabular}

Only results achieving significance at the $P=0.05$ level are shown. median postoperative visual acuity was 0.48 (equivalent to $6 / 18$ ) and the median improvement in acuity was 0.78 $\log$ MAR units.

For patients with no pre-existing cause for impaired acuity $48 \%$ regained $6 / 12$ Snellen or better $(52.1 \%$ if primary successful reattachment was also achieved) and the median improvement in acuity was $0.82 \log M A R$.

\section{Statistically significant factors predicting visual outcome}

For patients with no pre-existing reason for poor visual acuity, using a multiple variable model, the only statistically significant factor predictive of good final visual acuity was preoperative visual acuity $(P=0.001)$, with borderline associations with duration of macular detachment $(P=0.052)$ and grade of surgeon $(P=0.06)$. The duration of macular detachment ranged from less than 1 day to 28 months (median 9 days) - the accuracy of which was dependent on the reliability of patients' recollection. In 22 patients, it was not possible to date the onset of macular detachment. For patients with primary success and no pre-existing reason for poor vision and macular detachment of 1 week or less, 1 week to 2 weeks, 2-4 weeks and greater than 4 weeks, the mean visual acuities achieved were logMAR 0.47, 0.51, 0.74, and 0.59 , respectively. The paradoxically better outcome of patients with duration of macular detachment greater than 4 weeks (compared to 2-4 weeks) was because of a number of patients with relatively good preoperative visual acuities in the long duration group with shallow macular detachments. The influence of duration was therefore weak and a more important predictor of visual outcome was preoperative visual acuity so that with the same criteria, the visual outcomes of patients with preoperative visual acuity of $6 / 12$ or less, $6 / 18-6 / 60$, and worse than $6 / 60$ were mean visual acuities of logMAR 0.10, 0.33, and 0.59, respectively (equivalent to Snellen 6/7.5, 6/12.8, and $6 / 23.3$ ).

A breakdown of primary anatomical success rates for different types of surgery is shown in Table 4 .

Table 4 Operation types and success rates

\begin{tabular}{lcl}
\hline Operation type & $\begin{array}{c}\text { \% Primary success } \\
(\text { number successful/n) }\end{array}$ & Reasons for primary failures where this could be determined \\
\hline Buckle & $76.0 \%(35 / 46)$ & Three new/missed holes, 5 unsupported break, $2 \mathrm{PVR}$ \\
Vitrectomy/gas tamponade & $86.1 \%(93 / 108)$ & Two unsupported $/$ untreated, 5 new $/ \mathrm{missed} \mathrm{holes,} 7 \mathrm{PVR}$ \\
Vitrectomy with oil & $34.6 \%(9 / 26)$ & One PVR, oil at final follow-up in 10 \\
$\begin{array}{l}\text { Drain, air, cryo explant } \\
\text { (DACE) }\end{array}$ & $60 \%(3 / 5)$ & One unsupported break, 1 missed $/$ new break
\end{tabular}




\section{Discussion}

A National Audit in the United Kingdom in $2002^{5}$ reported on outcomes of retinal detachment surgery. In a subgroup of patients $(n=374)$ managed by specialist vitreoretinal surgeons there was an $82 \%$ primary reattachment rate for a varied case mix including macula-on and macula-off retinal detachments, using conventional scleral buckling (with or without external drainage) in $67 \%$. Further subgroups were defined on the basis of extent of detachment or features increasing the complexity of the case. Higher grades had worse primary anatomical success as expected. Whether patients with oil still in situ may have contributed to the higher primary success rate of vitreoretinal specialists (defined simply as a flat retina at three months) was not mentioned. In our study of 185 cases of purely macula-off retinal detachment but with an otherwise varied case mix, a primary anatomical success rate of $76 \%$ was achieved, with 10 patients added to the failure group because although their retinas were flat they still had oil in situ. Other studies have been criticized for including such patients in the primary success rate. ${ }^{6}$ In our practice, we estimate that only around $50 \%$ of patients with oil in situ will eventually have the oil removed. Others have found that $20 \%$ of these will have a recurrence of retinal detachment. ${ }^{7-9}$

Other relevant studies with the exception of that of Sullivan $e a^{1}$ relate to buckling surgery from the early 1980s and conventional surgery was used in 101 of 153 $(66 \%)$ of Sullivan's patients. In our study conventional surgery was used in only $30 \%$.

Our study has found that good preoperative logMAR visual acuity and the absence of PVR (grade C) predicted better primary anatomical success. This is a consistent feature of other studies. ${ }^{3,10}$ Patients with two or more retinal breaks had worse primary success rates than those with less.

Poor vision preoperatively may reflect a more bullous detachment ${ }^{10}$ with more elevated breaks, which could increase primary failure. ${ }^{1}$ Our study provides no evidence of any association between primary success and each of grade of surgeon, duration of macular detachment, lens status, age of patient and number of quadrants detached but this may reflect a lack of statistical power. Macular detachment of more than 30 days did, however, reduce ultimate success, as did a greater number of quadrants detached (the latter with univariate analysis but not significant with the multiple variable analysis). However, analysis of ultimate anatomical success is more complicated because of the range of surgical techniques available and the use of oil. Some other studies (where scleral buckling without vitrectomy was the surgical technique) also identify greater extent of retinal detachment ${ }^{3,11}$ and aphakia ${ }^{3,12}$ as factors reducing success.

A multiple variable analysis was carried out to determine preoperative factors predicting visual outcome, specifically in patients undergoing surgery for macula-off retinal detachment and with no other coexisting reason for poor vision. Good preoperative visual acuity was found to be the only factor strongly predictive of good visual outcome in these patients ( $P=0.001$ on multivariate analysis). This is in keeping with other studies. ${ }^{3,13-15}$ Friberg and Eller ${ }^{15}$ found that stronger correlation can be found if a potential acuity meter is used to measure preoperative vision, perhaps because this removes the confounding effect of the refractive error induced by the macular detachment or because less light scatter occurs. Factors associated with poor preoperative vision that are associated with poor postoperative visual outcome in other studies are height of foveal detachment ${ }^{16}$ and PVR. ${ }^{11,14}$ Increased height of the retinal detachment may be associated with more degeneration of the photoreceptors. ${ }^{17}$ However, in our study preoperative PVR was not associated with poor postoperative vision with univariate or multivariate analysis and height of the retina was not studied.

The age range was wide in this study (range 17-91 years, median 63). Younger patients may have better visual outcome, ${ }^{3}$ but there was no such association found in this study. Longer follow-up over years may be necessary to demonstrate gradual improvement in visual function that may be more likely with younger age. ${ }^{18}$

In patients without other coexisting ocular pathology, visual outcome following reattachment of macula-off retinal detachments may be limited by cystic outer retinal degeneration, cystoid macular oedema, epiretinal membrane, or localized subretinal fluid. ${ }^{19}$ Studies of visual recovery after macula-off retinal detachment have shown that $37-71 \%$ of patients with primary reattachment achieve $6 / 12$ or better. ${ }^{13,20-23}$ However, many of these studies excluded patients with long duration of macular detachment, coexisting pathology, PVR and any ocular disease such as diabetic retinopathy, macular degeneration or glaucoma. One study of vitrectomy (which excluded PVR grade C or worse) achieved $86 \%$ primary success in 178 patients with pseudophakic macula-off detachments without the use of oil. The median visual acuity achieved was $6 / 12 .^{22}$ In our study, which included a varied case mix and included patients with PVR grade C or worse $44 \%$ of patients achieved 6/12 (logMAR 0.3) or better with a median postoperative best visual acuity of $\log$ MAR 0.48 equivalent to Snellen 6/18.

Studies have suggested that the duration of macular detachment should be limited to a maximum of 7-9 days if possible after which the visual results of successful 
primary reattachment will be adversely affected. 3,14,20,21,24 However, these studies used almost entirely scleral buckling without vitrectomy. There is conflict between studies involving vitrectomy for retinal detachment as to whether duration of macular detachment is ${ }^{13}$ or is not ${ }^{22}$ a significant predictor of visual outcome. Height of macular detachment may be a relatively more important influence on visual recovery and may be a confounding factor when the effect of duration on visual outcome is being considered. This may explain why duration is a relatively weak risk factor when height of foveal detachment is not included in the analysis. In this study, the effect of duration almost reached statistical significance with multiple variable analysis $(P=0.052)$. We found seven examples of patients with greater than 10 weeks of macular detachment with $6 / 12$ or better visual outcome. Five of these seven patients had $6 / 36$ or better visual acuity preoperatively, which reinforces the suggestion that preoperative visual acuity is the more important factor predicting visual outcome.

Conversely some patients operated early and with primary anatomical success have poor visual outcome. ${ }^{21}$ Optical coherence tomography (OCT) has shown that increased height of foveal detachment may affect visual outcome with associated increased disruption of the retina, ${ }^{16,25}$ and that localized persisting subclinical submacular fluid may be associated with worse visual outcome when scleral buckles are used. ${ }^{19}$

In addition intraoperative drainage of subretinal fluid may adversely affect visual acuity, ${ }^{21,26}$ perhaps by a reperfusion injury although others have not found this effect. The effect of drainage was not analysed in our study because the patients were selected for different surgical techniques based on preoperative clinical features that would have biased outcomes.

The success rates for trainees can be high and may depend on the level of supervision and case selection. ${ }^{2,5}$ In this study there was no difference for different grades of surgeons in terms of primary anatomical success and both groups had similar visual outcomes.

Drawbacks of our study include multiple testing which increases the risk of type I statistical error and the retrospective nature of the study.

\section{Conclusion}

Macula-off retinal detachment surgery is a highly effective intervention. More than $75 \%$ of such detachments may be repaired with one operation and $44 \%$ of patients regain at least $6 / 12$ Snellen. The median $\log$ MAR acuity increment of 0.78 far exceeds that seen in cataract surgery.

This study of 185 patients with macula-off retinal detachment supports claims that preoperative visual acuity is the most important factor predicting final visual acuity, primary anatomical success, and ultimate anatomical success. We also find supporting evidence for the adverse effects of PVR, duration of macular detachment, number of breaks, and extent of detachment on anatomical success.

\section{References}

1 Sullivan PM, Luff AJ, Aylward GW. Results of primary retinal reattachment surgery: a prospective audit. Eye 1997; 11: 869-871.

2 Comer MB, Newman DK, George ND, Martin KR, Tom BDM, Moore AT. Who should manage primary retinal detachments? Eye 2000; 14: 572-578.

3 Laatikainen L, Harju H, Tolppanen EM. Post-operative outcome in rhegmatogenous retinal detachment. Acta Ophthalmologica 1985; 63: 647-655.

4 Minihan M, Tanner V, Williamson TH. Primary rhegmatogenous retinal detachment: 20 years of change. Br J Ophthalmol 2001; 85: 546-548.

5 Thompson JA, Snead MP, Billington BM, Barrie T, Thompson JR, Sparrow JM. National audit of the outcome of primary surgery for rhegmatogenous retinal detachment. I and II. Eye 2002; 16: 766-777.

6 Sullivan PM, Aylward GW. Letter to the editor. Eye 1989; 12: 750-751.

7 Unlü N, Kocaoglan H, Acar MA, Sargin M, Aslan BS, Duman S. Outcome of complex retinal detachment surgery after silicone oil removal. Int Ophthalmol 2004; 25: 33-36.

8 Laidlaw DAH, Karia N, Bunce C, Aylward GW, Gregor ZJ et al. Is prophylactic 360-degree laser retinopexy protective? Risk factors for retinal redetachment after removal of silicone oil. Ophthalmology 2002; 109: 153-158.

9 Falkner CI, Binder S, Kruger A. Outcome after silicone oil removal. BrJ Ophthalmol 2001; 85: 1324-1327.

10 Tani P, Robertson DM, Langworthy A. Prognosis for central visual and anatomic reattachment in rhegmatogenous retinal detachment with macula detached. Am J Ophthalmol 1981; 92: 611-620.

11 Girard P, Karpouzas I. Pseudophakic retinal detachment: anatomic and visual results. Graefe's Arch Clin Exp Ophthalmol 1995; 233: 324-330.

12 Burton TC. Preoperative factors influencing anatomic success rates following retinal detachment surgery. Trans Am Ophthalmol Soc 1977; 83: 499.

13 Speicher M, Fu AD, Martin JP, Von Fricken MA. Primary vitrectomy alone for repair of retinal detachments following cataract surgery. Retina 2000; 20: 459-464.

14 Isernhagen RD, Wilkinson CP. Visual acuity after the repair of pseudophakic retinal detachments involving the macula. Retina 1989; 9: 15-21.

15 Friberg TR, Eller AW. Prediction of visual recovery after scleral buckling of macula-off retinal detachments. Am J Ophthalmol 1992; 114: 715-722.

16 Hagimura N. Optical coherence tomography of the neurosensory retina in rhegmatogenous retinal detachment. Am J Ophthalmoly 2000; 129: 186-190.

17 Aaberg TM, Machemer R. Retinal detachment. In: Garner A, Klintworth GK (eds). Pathobiology of Ocular Disease: A Dynamic Approach, Part B. Marcel Dekker: New York, 1982. 
18 Kusaka S, Toshino A, Ohashi Y, Sakaue E. Long-term visual recovery after scleral buckling fo macula-off retinal detachments. Japan J Ophthalmol 1998; 42: 218-222.

19 Wolfensberger TJ, Gonvers M. Optical coherence tomography in the evaluation of incomplete visual acuity recovery after macula-off retinal detachments. Graefe's Arch Clin Exp Ophthalmol 2002; 240: 85-89.

20 Burton TC. Recovery of visual acuity after retinal detachment involving the macula. Trans Am Ophthalmol Soc 1982; 80: 475-497.

21 Ross WH, Kozy DW. Visual recovery in macula-off rhegmatogenous retinal detachments. Ophthalmology 1998; 105: 2149-2153.

22 Campo RV, Sipperly JO, Sneed SR et al. Pars plana vitrectomy without scleral buckle for pseudophakic retinal detachments. Ophthalmology 1999; 106: 1811-1816.
23 Kreissig I. A Practical Guide to Minimal Surgery for Retinal Detachment. Thieme Publishing Group, Theime New York 2000 Chapter 8.16. 2: 231.

24 Hassan TS, Sarrafizadeh R, Ruby AJ, Garretson BR Kuczynski B, Williams GA. The effect of duration of macular detachment on results after the scleral buckle repair of primary, macula-off retinal detachments. Ophthalmology 2002; 109: 146-152.

25 Lecleire CA, Muraine M, Menard JF, Brasseur G. Predictive visual outcome after macula-off retinal detachment surgery using optical coherence tomography. Retina 2005; 25: $44-53$

26 Tornambe PE, Hilton GF. The retinal detachment study group. Pneumatic retinopexy. A multicentre controlled trial comparing pneumatic retinopexy with scleral buckling. Ophthalmology 1989; 96: 772-784. 